\title{
Subdiffusion in one-dimensional Hamiltonian chains with sparse interactions
}

\author{
Wojciech De Roeck ${ }^{1}$, Francois Huveneers ${ }^{2}$, and Stefano Olla ${ }^{2}$ \\ ${ }^{1}$ Instituut Theoretische Fysica, KU Leuven, 3001 Leuven, Belgium \\ ${ }^{2}$ Ceremade, UMR-CNRS 7534, Université Paris Dauphine, PSL Research \\ University, 75775 Paris cedex 16, France
}

February 23, 2020

\begin{abstract}
We establish rigorously that transport is slower than diffusive for a class of disordered one-dimensional Hamiltonian chains. This is done by deriving quantitative bounds on the variance in equilibrium of the energy or particle current, as a function of time. The slow transport stems from the presence of rare insulating regions (Griffiths regions). In manybody disordered quantum chains, they correspond to regions of anomalously high disorder, where the system is in a localized phase. In contrast, we deal with quantum and classical disordered chains where the interactions, usually referred to as anharmonic couplings in classical systems, are sparse. The system hosts thus rare regions with no interactions and, since the chain is Anderson localized in the absence of interactions, the non-interacting rare regions are insulating. Part of the mathematical interest of our model is that it is one of the few non-integrable models where the diffusion constant can be rigorously proven not to be infinite.
\end{abstract}

Dedicated to Joel L. Lebowitz, for being a constant source of inspiration

\section{Introduction}

One-dimensional Hamiltonian systems, also known as chains, sometimes exhibit anomalous transport properties, i.e. non-diffusive transport of locally conserved quantities like energy, particle number, momentum, etc. Whereas the possibility of superdiffusion is well-documented [30], subdiffusion has not been studied as intensively.

In disordered systems, Anderson localization can actually suppress transport entirely [6, $22,29,21]$. It can be realized e.g. in disordered chains of free fermions or, equivalently, in some quantum spin chains. Perhaps surprisingly, it can also be realized in classical disordered harmonic chains: The dynamics is linear and all the modes of the chain become exponentially 
localized in the presence of disorder. ${ }^{1}$ For Anderson insulators, the current of conserved quantities is exponentially suppressed as a function of the length of the chain and, in equilibrium, the variance of the current of a conserved quantity, $C(t)$, does not grow with time, i.e. $C(t)=\mathcal{O}(1)$ as $t \rightarrow \infty$ [10]. See (8) below for a precise definition of $C(t)$. For the quantum systems quoted above, there is strong evidence that, upon turning on interactions, localization persists at strong disorder and weak interactions, resulting in the so called many-body localized (MBL) phase [20, 24, 9, 39, 45, 27], see also [7] for reviews and more recent progress. There as well we expect $C(t)=\mathcal{O}(1)$ as $t \rightarrow \infty$. In contrast, for classical systems, arbitrarily small anharmonic interactions are expected to destroy localization $[18,40,37,8]$.

Subdiffusion refers to the case where conserved quantities do evolve at the macroscopic scale, i.e. $C(t) \rightarrow \infty$, but slow enough so that $C(t) / t \rightarrow 0$ as $t \rightarrow \infty$. Contrary to localization, subdiffusion does not challenge the basic principles of thermodynamics, and we may expect the phenomenon to be more widespread. Interestingly, the existence of a subdiffusive ergodic phase has been predicted theoretically $[3,23,2,41,5,31]$ to occur in quantum chains with moderate disorder, close below the MBL transition point, see Figure 1. This prediction has been verified numerically, although, to our best knowledge, it is not conclusively known whether the observed subdiffusion can unambiguously be ascribed to the theoretical predictions $[35,32,33,46,28,42$, $36,44]$. This phenomenon is purely one-dimensional and rests on the presence of bottlenecks in the chain ${ }^{2}$. For generic classical systems, one does not expect the existence of an analogous subdiffusive phase, see [8] for a detailed analysis.

In this paper, we present a mathematically rigorous derivation of slower-than-diffusive transport in a class of quantum and classical models, suggested to us by D. A. Huse. In contrast to most models studied in the literature, the interactions considered here are sparse (but not weak), turning them on at each site independently with probability $p<1$. A similar set-up was recently studied in [38] and mathematical results on long transmission times were derived. As it turns out, the existence of a subdiffusive phase can then be shown to hold both for classical and quantum Hamiltonians (even though the corresponding classical systems are still not expected to have a MBL phase).

The rest of this paper is organized as follows. Below, we provide a more detailed heuristic explanation of the phenomenology. In Section 2, we define properly the classcial and quantum Hamiltonians studied in this paper, and we state precise conditions for the existence of a subdiffusive phase in Theorem 1 for the classical chain, and in Theorem 2 for the quantum chain. The proof of Theorem 1 is presented in Section 3 and the proof of Theorem 2 in Section 4.

\subsection{Griffiths regions in disordered chains}

Following [3], let us explain heuristically the origin of subdiffusion in disordered quantum systems. For the sake of concreteness, let us consider the celebrated "disordered XXZ spin chain":

$$
H=\sum_{x=1}^{L-1}\left(J\left(S_{x}^{(1)} S_{x+1}^{(1)}+S_{x}^{(2)} S_{x+1}^{(2)}\right)+g S_{x}^{(3)} S_{x+1}^{(3)}\right)+\sum_{x=1}^{L} \omega_{x} S_{x}^{(3)}
$$

\footnotetext{
${ }^{1}$ The first instances of disordered harmonic chains have been introduced in $[43,12]$. Because of momentum conservation, the localization length diverges in the bottom of the spectrum for these models, yielding a more complicated phenomenology. We will not deal with such cases here.

${ }^{2}$ More precisely, it rests on the sparsity of loops and the presence of bottlenecks, see [17].
} 
which has become the standard model for MBL. Here, $S_{x}^{1,2,3}$ are the spin- $\frac{1}{2}$ spin operators (Pauli matrices) acting on site $x$ of a chain (see e.g. [4] for more background and a full definition) and $J, g$ are parameters of the model. The on-site fields $\omega_{x}$ are assumed to be independent, identically distributed (iid) random variables, e.g. distributed according to a normal distribution with zero mean and standard deviation $W$, so that the parameter $W$ plays the role of the "disorder strength". The specific choice of the distribution plays no crucial role, and in particular the fact that $\omega_{x}$ is unbounded turns out to be eventually irrelevant. For $g=0$, the system is an Anderson insulator, since it can be mapped to free fermions by a Jordan-Wigner transformation, see e.g. [1]. Hence, it is localized at all values of $W>0$. Instead $g>0$ brings in interactions among the spins and the system is expected to stay localized only at large disorder $W>W_{c}$, while it becomes delocalized for smaller values of $W$, see Figure 1.

For $W>W_{c}$, i.e. in the localized phase, the resistance of of a stretch of length $\ell$ is given by

$$
r \sim \mathrm{e}^{2 \ell / \xi}
$$

where $\xi=\xi(W)$ is the localization length characterizing the localized system. Strictly speaking, the resistance $r$ depends on the disorder realization, but for large $\ell$, we can neglect the fluctuations around the average value in (2). Here, we focus on a system where the disorder strength is actually too small to bring about localization, i.e. $W<W_{c}$, but local anomalously large disorder values can be strong enough to put small stretches of the systems in the localized phase, i.e. these regions would be in the localized phase if they would be disconnected from the rest of the chain. For example, it can be that on a stretch of size $\ell$, the value of the empirical standard deviation $\tilde{W}$ is larger than $W_{c}$. Such a region is called a Griffiths region. As can be inferred from the previous example, a Griffiths region of length $\ell$ occurs in a given place with probability $\mathrm{e}^{-c(W) \ell}$, where the large deviation rate $c(W)$ is expected to vanish as $W$ approaches the critical value $W_{c}$. If we assume for simplicity that all such Griffiths regions have the same localization length $\xi_{*}$, then the probability distribution of the resistance $r$ of a single Griffiths region is given roughly by

$$
p(r) \mathrm{d} r \propto \mathrm{e}^{-c(W) \ell} \mathrm{e}^{-2 \ell / \xi_{*}} \mathrm{~d} r \propto r^{-\left(1+\xi_{*} c(W) / 2\right)} \mathrm{d} r .
$$

For $\xi_{*} c(W)<2$, we notice that $E(r)=\infty$.

To understand how Griffiths regions can lead to a subdiffusive behavior when $\xi_{*} c(W)<2$, we represent the chain as a Ohmic circuit of resistances $\left(r_{i}\right)_{1 \leq i \leq L}$ in series, where the resistances are the Griffiths regions (sites outside of any Griffiths region may simply be assumed to have a resistance of order 1 ). The resistances $\left(r_{i}\right)_{1 \leq i \leq L}$ are i.i.d. in very good approximation. The total resistance is $R=\sum_{i=1}^{L} r_{i}$ and the conductivity, which is more suited for our discussion, is given by $K=L / R$, where $L$ stands for the length of the chain. If the resistances $r_{i}$ have very heavy tails, in particular if $E\left(r_{i}\right)=\infty$, then the conductivity vanishes almost surely: $K \rightarrow 0$, as $L \rightarrow \infty$. Instead, if $E\left(r_{i}\right)<\infty$, then $K>0$. The system is thus subdiffusive if $\xi_{*} c(W)<2$, i.e. if one is not too far from the localized phase, see Figure 1. 


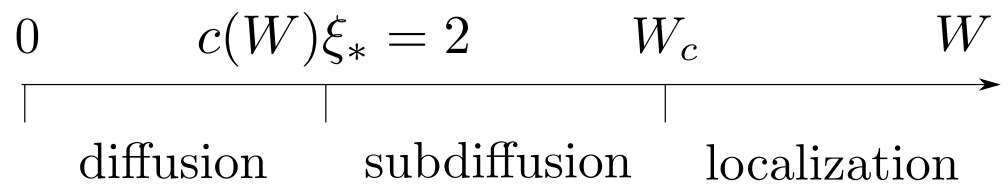

Figure 1: Transport in a generic quantum chain as a function of disorder strength $W$.

\subsection{Systems without genuine localized phase}

As said, for classical systems with generic anharmonic interactions, one does not expect a genuinely localized phase. This is related to the fact that, at positive temperature in the thermodynamic limit, all typical configurations are expected to contain some chaotic spots that will eventually break the quasi-periodic behavior of the dynamics. Yet, the dynamics in certain classical systems can certainly be extremely slow and glassy, see [16] for a recent review. The biggest difference with quantum chains is that the presence of an insulating Griffiths region is mainly singled out by an atypical configuration of the oscillators. Indeed, large disorder fluctuations will only ensure that a larger proportion of the configurations is insulating, never all of them in generic cases. Therefore, if the dynamics is eventually ergodic as one expects, a specific Griffiths region exists only for a finite time. A detailed quantitative analysis done in [8] shows that the lifetime of Griffiths regions, compared to their resistance, is too short to bring about subdiffusion. While this conclusion pertains to generic anharmonic disorder chains of oscillators, we will see that it does not apply to the more specific class of classical Hamiltonians considered in this paper.

\subsection{Sparse interactions}

The models treated in this paper have the following specific property: Interactions or anharmonic couplings are present at each site with probability $p$ and absent with probability $1-p$. In a typical realization, there are hence arbitrarily long stretches of sites with no interaction and these stretches play the role of Griffiths regions. These Griffiths regions are thus simply Anderson insulators. In particular, the issue raised above for classical chains is avoided: All the modes of the chain are exponentially localized and the region behaves as a perfect insulator (i.e. with a conductivity vanishing exponentially with the length) independently of the region in phase space that the system occupies.

Finally, one may wonder whether our quantum model is actually localized in the regime where we predict subdiffusion. The rigorous treatment of this question goes way beyond the present paper, but we can speculate on the phase diagram of our model. Let us restrict to $p \ll 1$, i.e. very sparse interactions. In the regions where the interactions are switched off, our model is simply an Anderson insulator and hence localized regardless of the disorder strength. Then, the question is whether the sparse regions with interaction can delocalize such a system. This question has been investigated and answered in previous work, [15, 34], and the upshot is that we should expect the system to be localized whenever the localization length of the non-interacting system is small enough, and delocalized otherwise. Therefore, our model is indeed expected to have a localization-delocalization transition and the region where we prove subdiffusion overlaps 
with presumed delocalized phase.

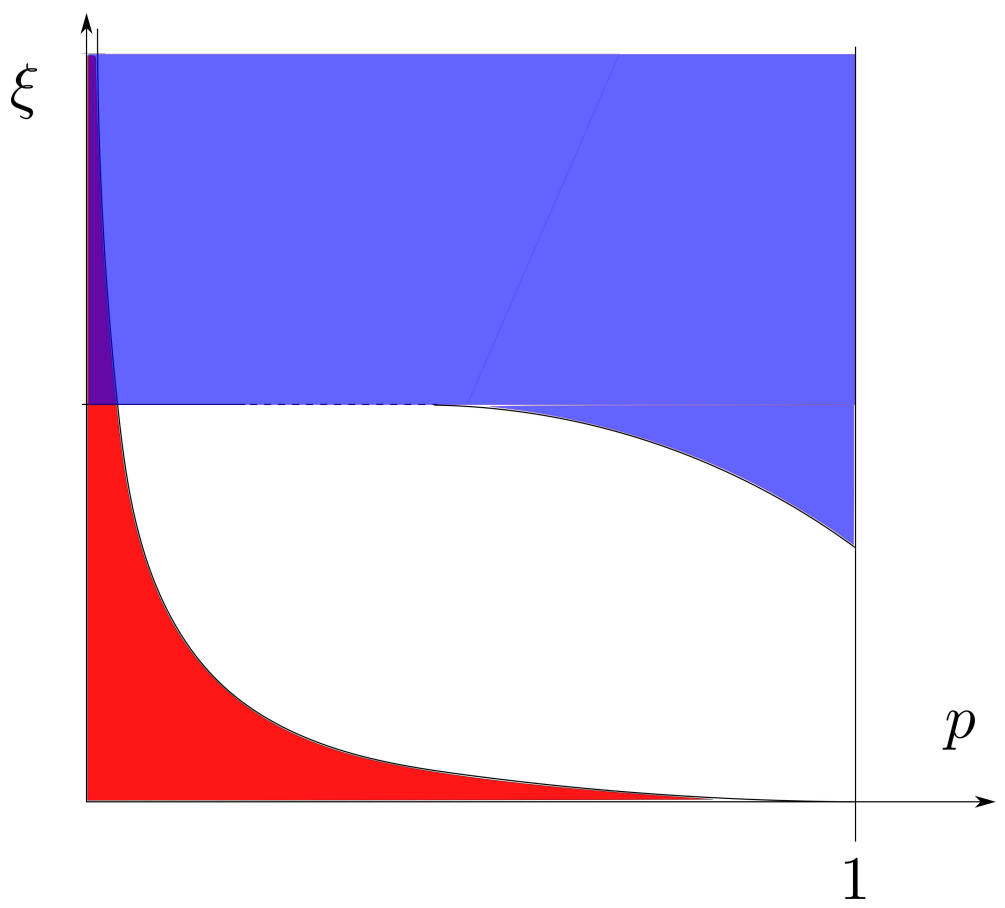

Figure 2: Our proof of slower than subdiffusive transport applies in the red area of the $p-\xi$ plane. For our quantum model, the blue area indicates where we expect a delocalized (non-MBL) phase, and we see that these regions have a non-zero intersection

\section{Models and Results}

We study in details two different Hamiltonian chains. Each of them is representative of a larger class of systems. Let $\mathbb{N}_{L}=\{1, \ldots, L\}$ be the set of sites, and we will eventually take the length $L \rightarrow \infty$.

\subsection{Anharmonic disordered chain}

Let us first consider the disordered classical anharmonic chain, described by the following Hamiltonian on $\mathbb{R}^{2 L}$ :

$$
H(q, p)=\sum_{x=1}^{L} h\left(q_{x}, q_{x+1}, p_{x}, \omega_{x}, \tau_{x}\right)=\sum_{x=1}^{L}\left(\frac{p_{x}^{2}}{2}+\omega_{x}^{2} \frac{q_{x}^{2}}{2}+g \tau_{x} \frac{q_{x}^{4}}{4}+g_{0} \frac{\left(q_{x+1}-q_{x}\right)^{2}}{2}\right)
$$

with Neumann boundary conditions at both ends, i.e. $q_{L+1}=q_{L}$. We take the anharmonic coupling $g$ and the harmonic coupling $g_{0}$ to be non-negative.

The frequencies of the uncoupled oscillators, $\left(\omega_{x}\right)_{x \in \mathbb{N}_{L}}$, are independent and identically distributed (iid) random variables with a bounded density with compact support not containing 0 , 
i.e. there exists $\omega_{-}>0$ such that

$$
\omega_{x}^{2} \geq \omega_{-}^{2}>0 \quad \text { almost surely. }
$$

The latter condition is most likely not essential but guarantees that the Hamiltonian $H$ is strictly convex, a feature that turns out to be convenient to establish the decay of correlations in the Gibbs ensemble. The variables $\tau_{x}$ are iid random variables, independent of $\left(\omega_{x}\right)$, and with value in $\{0,1\}$. We define

$$
p=P\left(\tau_{x}=1\right) .
$$

The dynamics is given by Hamilton's equations:

$$
\dot{q}=p, \quad \dot{p}=-\nabla_{q} H(q, p)
$$

and the generator of the dynamics is given by the usual Liouville operator:

$$
\mathcal{L}=p \cdot \nabla_{q}-\nabla_{q} H \cdot \nabla_{p}
$$

The energy is the only obvious locally conserved quantity. For simplicity let us write $h_{x}=$ $h\left(q_{x}, q_{x+1}, p_{x}, \omega_{x}, \tau_{x}\right)$. For $x \in \mathbb{N}_{L}$, we define the energy current $j_{x}$ between the site $x$ and $x+1$ as

$$
j_{x}=\left\{h_{x+1}, h_{x}\right\}=-g_{0} p_{x+1}\left(q_{x+1}-q_{x}\right)
$$

with the conventions $q_{0}=q_{1}$ and $q_{L+1}=q_{L}$, so that $j_{0}=j_{L}=0$ as imposed by the Neumann boundary conditions in (3). This definition ensures that the currents satisfy the continuity equation

$$
\mathcal{L} h_{x}=-\nabla j_{x}=j_{x-1}-j_{x}
$$

\subsubsection{The harmonic chain: $g=0$}

At $g=0$, the oscillator chain is an Anderson insulator due to disorder. Indeed, the equations of motion (4) are linear and the equation for $p$ takes the form

$$
\dot{p}_{x}=-\sum_{y} \mathcal{H}_{x, y} q_{y} \quad \text { with } \quad \mathcal{H}=V-g_{0} \Delta
$$

where $V_{x, y}=\omega_{x}^{2} \delta_{x, y}$ and where $\Delta$ is the discrete laplacian on $\mathbb{N}_{L}:=\{1, \ldots, L\}$ with Neumann boundary conditions:

$$
\Delta f(x)=f(x-1)-2 f(x)+f(x+1) \quad \text { for } \quad x \in \mathbb{N}_{L}
$$

with the convention $f(0)=f(1)$ and $f(L+1)=f(L)$. The random operator $\mathcal{H}$ on $l^{2}\left(\mathbb{N}_{L}, \mathbb{R}\right)$ is known as the Anderson Hamiltonian for a single quantum particle [6]. It is a celebrated result $[22,29]$ that its eigenvectors are localized, see $[13,14,19]$ for pedagogical expositions.

Lemma 1 (Anderson localization, Theorem IX.1 in [29]). Let $\left(\psi_{k}\right)_{1 \leq k \leq L}$ be an orthonormal basis of eigenvectors of $\mathcal{H}$ (depending hence on the realization of $\left(\omega_{x}\right)$ ). There are $\mathrm{C}<+\infty$ and $\xi>0$, such that, for any $L$ and $1 \leq x, y \leq L$,

$$
E\left(\sum_{k=1}^{L}\left|\psi_{k}(x) \psi_{k}(y)\right|\right) \leq \mathrm{Ce}^{-|x-y| / \xi}
$$


(since we assume that the distribution of $\omega_{x}$ is compactly supported, we may take $A=\mathbb{R}$ in Theorem IX.1 in [29]). We refer to $\xi$ as the localization length of the system, though it is more precisely an upper bound for the localization length uniform in the energy. We note that the localization length $\xi$ depends thus only on $g_{0}$ and on the distribution of $\left(\omega_{x}\right)$.

\subsubsection{The anharmonic chain: $g \geq 0$}

We finally denote by $\langle\cdot\rangle_{\beta}$ the expectation with respect with the Gibbs measure at inverse temperature $\beta>0$, i.e. the probability measure on $\mathbb{R}^{2 L}$ with density

$$
\rho_{\beta}(q, p)=\frac{\mathrm{e}^{-\beta H(q, p)}}{Z(\beta)}
$$

where $Z(\beta)$ ensures the normalization (the partition function). Note that $\left\langle j_{x}\right\rangle_{\beta}=0$ for any $x$, because the currents $j_{x}$ are odd in the $p$-variables. The thermal conductivity $\kappa$ at inverse temperature $\beta$ is defined by

$$
\kappa=\beta^{2} \lim _{t \rightarrow \infty} \frac{C(t)}{t} \quad \text { with } \quad C(t)=\limsup _{L \rightarrow \infty}\left\langle\left(\int_{0}^{t} \mathrm{~d} s \frac{1}{\sqrt{L}} \sum_{x=1}^{L-1} j_{x}(s)\right)^{2}\right\rangle_{\beta} .
$$

The expression for $C(t)$ is well-defined because we view $\left(\omega_{x}, \tau_{x}\right)_{x \in \mathbb{N}_{L}}$ as restrictions of a single infinite array $\left(\omega_{x}, \tau_{x}\right)_{x \in \mathbb{N}}$ of i.i.d. random variables, so that $C(t)$ is a limit superior of random variables defined on a single probability space. Furthermore, in the definition of $C(t)$, we expect that the limit superior is actually a limit, almost surely, but we are not able to prove this. This is however irrelevant for establishing that transport is slower than diffusive, which is equivalent to the fact that $C(t) / t \rightarrow 0$ almost surely, as $t \rightarrow \infty$. In Theorem 1 below, we provide an upper bound on $C(t)$ implying slower than diffusive behavior of the transport for sufficiently small $p$. We recall that $C(t)=\mathcal{O}(f(t)), t \rightarrow \infty$ means that $\frac{C(t)}{f(t)}$ is bounded as $t \rightarrow \infty$.

Theorem 1. Fix any $\beta>0, g \geq 0, g_{0}>0$. If the inequality

$$
\gamma:=\frac{4}{1+\left(3 \xi \log \left(\frac{1}{1-p}\right)\right)^{-1}}<1
$$

holds (i.e. if $p<1-\mathrm{e}^{-1 / 9 \xi}$ ), with $\xi$ the localization length given in (6), then

$$
C(t)=\mathcal{O}\left((\log t)^{5} t^{\gamma}\right), \quad t \rightarrow \infty, \quad \text { almost surely }
$$

Remark 1. For $p=0$ (equivalently $g=0$ ) our bound gives $\gamma=0$. However, in that case the dynamics is localized and it is known that $C(t)=\mathcal{O}(1)$ as $t \rightarrow \infty$, see the remarks below Theorem 1 in [10].

Remark 2. By direct adaptation of the proof, the on-site potential $q \mapsto g q_{x}^{4}$ may be replaced by a more general potential $q \mapsto V\left(\theta_{x} q\right)$, where $V$ is a smooth, convex, local function, growing at most polynomially at infinity as well as all its derivatives, and where $\theta_{x} q$ denotes the shifted configuration by $x$. 


\subsection{Disordered Fermion chain}

This model differs from the one discussed above because it is quantum and, more importantly, it is believed to have a genuinely localized interacting phase (MBL) for some range of parameters.

To define the fermion chain, we need the fermionic creation and annihilation operators $c_{x}^{\dagger}, c_{x}$ that satisfy the canonical anticommutation relations

$$
\left[c_{x}, c_{x^{\prime}}^{\dagger}\right]_{+}=\delta_{x, x^{\prime}}, \quad\left[c_{x}, c_{x^{\prime}}\right]_{+}=\left[c_{x}^{\dagger}, c_{x^{\prime}}^{\dagger}\right]_{+}=0
$$

where $[a, b]_{+}=a b+b a$ is the anticommutator and $x, x^{\prime} \in \mathbb{N}_{L}$. We refer again to standard texts, e.g. [4], for an explicit representation of $c_{x}, c_{x}^{\dagger}$ as operators on the appropriate finite-dimensional Hilbert space $\mathcal{F}_{L}$. We also introduce the fermionic number operators $n_{x}=c_{x}^{\dagger} c_{x}$ counting the number of fermions ( 0 or 1 ) at site $x$. The Hamiltonian models interacting spinless fermions:

$$
H=\sum_{x=1}^{L-1}\left(J\left(c_{x}^{\dagger} c_{x+1}+c_{x+1}^{\dagger} c_{x}\right)+g \tau_{x} n_{x} n_{x+1}\right)+\sum_{x=1}^{L} \omega_{x} n_{x}
$$

where the random variables $\omega_{x}, \tau_{x}$ have the same law as those in Section 2.1.

This model can be mapped by the Jordan-Wigner transformation to a disordered variant of the XXZ-model:

$$
H=\sum_{x=1}^{L-1}\left(J\left(S_{x}^{(1)} S_{x+1}^{(1)}+S_{x}^{(2)} S_{x+1}^{(2)}\right)+g \tau_{x} S_{x}^{(3)} S_{x+1}^{(3)}\right)+\sum_{x=1}^{L} \omega_{x} S_{x}^{(3)} .
$$

In particular, setting $\tau_{x}=1$ yields back the disordered XXZ chain introduced in (1). For reasons of convenience, we work however with the fermionic chain.

\subsubsection{Dynamics}

In general, by observables, we mean any Hermitian operator $a$ acting on $\mathcal{F}_{L}$. The dynamics of observables is given by the Heisenberg evolution equation

$$
\frac{\mathrm{d}}{\mathrm{d} t} a_{t}=\mathcal{L} a_{t}:=\mathrm{i}[H, a], \quad a_{0}=a
$$

The model, has two conserved quantities, the energy, i.e. the Hamiltonian itself and particle number $N=\sum_{x} n_{x}$. Indeed, one checks easily that $[H, N]=0$. For simplicity, we discuss only transport of the particle number. The natural particle current operator $j_{x}$ is defined by

$$
j_{x}=\mathrm{i}\left[J\left(c_{x}^{\dagger} c_{x+1}+c_{x+1}^{\dagger} c_{x}\right), n_{x}\right]=\mathrm{i} J\left(c_{x+1}^{\dagger} c_{x}-c_{x}^{\dagger} c_{x+1}\right)
$$

and it satisfies the continuity equation

$$
\mathcal{L} n_{x}=-\nabla j_{x}=j_{x-1}-j_{x} .
$$

As for the equilibrium state of the system, we choose the density matrix $\rho_{\mu} \propto \mathrm{e}^{-\mu N}$. The expectation value of observables in this state is then given by

$$
\langle a\rangle_{\mu}=\frac{\operatorname{Tr}\left(\rho_{\mu} a\right)}{\operatorname{Tr}\left(\rho_{\mu}\right)}
$$

where $\operatorname{Tr}(\cdot)$ is the trace over the Hilbert space $\mathcal{F}_{L}$. Just as in Section 2.1, there are no subtle mathematical issues here because our setup is at finite volume. Note finally that $\left\langle j_{x}\right\rangle_{\mu}=0$. This follows for example by considering the gauge transformation $c_{x} \rightarrow \mathrm{e}^{\mathrm{i} \theta_{x}} c_{x}, c_{x}^{\dagger} \rightarrow \mathrm{e}^{-\mathrm{i} \theta_{x}} c_{x}^{\dagger}$, under which the state $\langle\cdot\rangle_{\mu}$ is invariant. 


\subsubsection{The non-interacting chain}

When $g=0$, the Hamiltonian reduces to

$$
H=\sum_{x=1}^{L-1} J\left(c_{x+1 c_{x}}^{\dagger}+c_{x}^{\dagger} c_{x+1}\right)+\sum_{x=1}^{L} \omega_{x} n_{x}=\sum_{x, x^{\prime} \in \mathbb{N}_{L}} c_{x^{\prime}}^{\dagger} \widetilde{\mathcal{H}}_{x, x^{\prime}} c_{x}
$$

where

$$
\widetilde{\mathcal{H}}=J \Delta+2 J+V
$$

with $V_{x, y}=\omega_{x} \delta_{x, y}$, is again the Anderson Hamiltonian. It only differs from $\mathcal{H}$ of Section 2.1 by irrelevant constants and a different distribution of on-site disorder. Obviously, Lemma 1, formulated for $\mathcal{H}$, applies to $\widetilde{\mathcal{H}}$ just as well and it yields an upper bound $\xi$ for the localization length that depends only on $J$ and on the distribution of $\omega_{x}$. The equality in (12) means that the Hamiltonian $H$ is the second quantization of $\widetilde{\mathcal{H}}$, or, in more intuitive terms, that $H$ describes no-interacting fermions.

Just like in the classical anharmonic chain, we consider the current-current correlation

$$
C(t)=\limsup _{L \rightarrow \infty}\left\langle\left(\int_{0}^{t} \mathrm{~d} s \frac{1}{\sqrt{L}} \sum_{x=1}^{L-1} j_{x}(s)\right)^{2}\right\rangle_{\mu} .
$$

The slower than diffusive behavior of the dynamics is equivalent to the fact that $C(t) / t \rightarrow 0$ as $t \rightarrow \infty$. In Theorem 2 below, we provide an upper bound on $C(t)$ guaranteeing slower than diffusive dynamics for sufficiently small $p$.

Theorem 2. Fix any $\mu>0, g \geq 0, J>0$. If the inequality

$$
\gamma:=\frac{4}{1+\left(3 \xi \log \left(\frac{1}{1-p}\right)\right)^{-1}}<1
$$

holds (i.e. if $p<1-\mathrm{e}^{-1 / 9 \xi}$ ), with $\xi$ the localization length given in (6), then the dynamics is slower than diffusive:

$$
C(t)=\mathcal{O}\left((\log t)^{5} t^{\gamma}\right), \quad t \rightarrow \infty, \quad \text { almost surely }
$$

\section{Proof of Theorem 1}

For $p=0$, the chain is harmonic and it is known that $C(t)=\mathcal{O}(1)$ as $t \rightarrow \infty$ (see the remarks below Theorem 1 in [10]). From now on, we assume $p>0$, hence the model is non-integrable. Throughout the proof, constants $\mathrm{C}$ can always depend on $\beta, g, g_{0}$ and the distribution of $\omega_{x}$ (unless stated otherwise), but not on the length $L$.

\subsection{Decay of static correlations}

We need decay of spatial correlations in the Gibbs state $\langle\cdot\rangle_{\beta}$. Given a function $u$ on $\mathbb{R}^{2 L}$, we denote by $\operatorname{supp}(u) \subset \mathbb{N}_{L}$ the smallest set of points such that $u$ is constant as a function of $q_{x}, p_{x}$ if $x \notin \operatorname{supp}(u)$ (it is thus not the usual support of a function in $\mathbb{R}^{2 L}$ ). Given two functions $u, v$, 
we denote by $d(u, v) \geq 0$ the distance between $\operatorname{supp}(u)$ and $\operatorname{supp}(v)$. If $u$ denotes a smooth function on $\mathbb{R}^{2 L}$, we write

$$
\nabla u=\left(\nabla_{q} u, \nabla_{p} u\right)=\left(\left(\partial_{q_{x}} u\right)_{1 \leq x \leq L},\left(\partial_{p_{x}} u\right)_{1 \leq x \leq L}\right) .
$$

The Lemma below yields the needed decay of correlations; it is a special case of more general results stated in $[25,26]$, see also [11] for a review.

Lemma 2 (decay of correlations). 1. Given $r \in \mathbb{N}$, there exists $\mathrm{C}_{r}<+\infty$ such that, for any $1 \leq x \leq L$,

$$
\left|\left\langle q_{x}^{r}\right\rangle_{\beta}\right| \leq \mathrm{C}_{r}, \quad\left|\left\langle p_{x}^{r}\right\rangle_{\beta}\right| \leq \mathrm{C}_{r} .
$$

2. There exist $\mathrm{C}, \zeta<+\infty$ such that, given two polynomials $u, v$ on $\mathbb{R}^{2 L}$ with $\langle u\rangle_{\beta}=\langle v\rangle_{\beta}=0$,

$$
\left|\langle u v\rangle_{\beta}\right| \leq \mathrm{Ce}^{-d(u, v) / \zeta}\langle\nabla u \cdot \nabla u\rangle_{\beta}^{1 / 2}\langle\nabla v \cdot \nabla v\rangle_{\beta}^{1 / 2}
$$

These bounds hold almost surely and the constants $\mathrm{C}, \mathrm{C}_{r}, \zeta$ are independent of $g$.

Proof. Polynomials are obviously integrable with respect to the measure $\mu_{\beta}$ at fixed length $L$. Let us start with Part 2, following Section 4 in [25]. We first notice that there is no need for the restriction $u=q_{i}$ or $u=p_{i}$ and $v=q_{j}$ or $v=p_{j}$ for some $1 \leq i, j \leq L$ in [25] (see also the statement of Theorem 3.1 in [11]). Next, the function

$$
(q, p) \mapsto \beta \sum_{x=1}^{L}\left(\frac{p_{x}^{2}}{2}+\omega_{x}^{2} \frac{q_{x}^{2}}{2}+g \tau_{x} \frac{q_{x}^{4}}{4}+g_{0} \frac{\left(q_{x+1}-q_{x}\right)^{2}}{2}\right)
$$

is strictly convex with a Hessian bounded below by $\beta \min \left(1, \omega_{-}^{2}\right)$. From there, the result follows as in [25], if $\zeta$ is taken large enough compared to $g_{0}$ and $g_{0} / \omega_{-}^{2}$. Let us next consider Part 1. We notice that $\left\langle p_{x}^{r}\right\rangle_{\beta}=(\beta / 2 \pi)^{1 / 2} \int_{\mathbb{R}} y^{r} \mathrm{e}^{-\beta y^{2} / 2} \mathrm{~d} y$, so that we only need to deal with the bound on $\left\langle q_{x}^{r}\right\rangle_{\beta}$. It is enough to consider $r$ even since $\left\langle q_{x}^{r}\right\rangle_{\beta}=0$ otherwise. For $r=2$, the result follows from part 2 of the Lemma taking $u=v=q_{x}$. For $r>2$, we obtain similarly $\left\langle q_{x}^{r}\right\rangle_{\beta} \leq \mathrm{C}\left\langle q_{x}^{r-2}\right\rangle_{\beta}$, and the result is obtained recursively.

\subsection{Splitting of the harmonic Hamiltonian}

We now exploit the fact that the system is an Anderson insulator in the regions where the anharmonic potential is absent, i.e. where $\tau=0$. The upshot is Lemma 3, whose significance is explained below. The material below is largely taken from [10], but we have tried to streamline the presentation to make the reasoning more transparant.

Throughout this section, we fix a realization of $\left(\tau_{x}\right)_{x \geq 1}$. Given $\ell \in \mathbb{N}$, let

$$
G_{0}(\ell)=\left\{x \in \mathbb{N}: \tau_{y}=0 \text { for all } y \in \mathbb{N} \text { s.t. }|y-x| \leq \ell\right\} .
$$

We also fix an element $x \in G_{0}(\ell)$ and we denote $B=\{x-\ell, \ldots, x+\ell\}$, such that $\tau_{y}=0$ for $y \in B$. Until the end of this section 3.2, the expectation $E$ is assumed to be conditioned on $\tau_{y}=0, y \in B$ and we do not repeat this.

In general, let $H_{X}$ be the Hamiltonian restricted to a finite set $X \subset \mathbb{N}$, i.e. retaining only terms in (3) whose support is in $X$. Similarly, we define the restriction of the Anderson operator $\mathcal{H}_{X}=\sum_{x, y \in X} \mathcal{H}_{x, y}$ and of the Liouville operator $\mathcal{L}_{\mathcal{X}}=\left\{\mathcal{H}_{\mathcal{X}}, \cdot\right\}$. 


\subsubsection{A priori left-right splitting}

We first consider an obvious splitting $H_{B}=H_{\mathrm{L}}+H_{\mathrm{R}}$ into a left $(\mathrm{L})$ and right $(\mathrm{R})$ part with respect to the midpoint $x$ :

$$
H_{\mathrm{L}}=H_{\{y \in B: y<x\}}, \quad H_{\mathrm{R}}=H_{B}-H_{\mathrm{L}}
$$

Note that hence $H_{\mathrm{R}}=H_{\{y \in B: y \geq x\}}+g_{0} \frac{1}{2}\left(q_{x}-q_{x-1}\right)^{2}$ The functions $H_{\mathrm{L}}, H_{\mathrm{R}}$ do not commute with $H_{B}$ in general, reflecting the fact that energy can be transported.

Our aim in this section is to find a modified left-right splitting $H_{B}=\widetilde{H}_{\mathrm{L}}+\widetilde{H}_{\mathrm{R}}$ which does satisfy the invariance property $\left\{H_{B}, \widetilde{H}_{\mathrm{L}, \mathrm{R}}\right\}=0$, reflecting the spatial localization of energy.

\subsubsection{An invariant splitting}

Let $\left(\psi_{k}\right)_{k \in \mathcal{I}}$ be an orthonormal basis of real eigenfunctions of $\mathcal{H}_{B}$, with $\mathcal{I}$ an index set, $|\mathcal{I}|=|B|$, and let $\left(\nu_{k}^{2}\right)$ be the corresponding eigenvalues, positive due to the positive-definitiveness of $\mathcal{H}$. For any $k \in \mathcal{I}$, the function

$$
e_{k}=\frac{1}{2}\left(\left\langle p, \psi_{k}\right\rangle^{2}+\nu_{k}^{2}\left\langle q, \psi_{k}\right\rangle^{2}\right)
$$

represents the energy of eigenmode $k$. The functions $e_{k}$ have two remarkable properties, following from standard considerations,

1. $\mathcal{L}_{B} e_{k}=0$ for all $k \in \mathcal{I}$.

2. $H_{B}=\sum_{k} e_{k}$.

The splitting $H_{B}=\widetilde{H}_{\mathrm{L}}+\widetilde{H}_{\mathrm{R}}$ that we propose is defined by

$$
\widetilde{H}_{\mathrm{L}}=\sum_{y} \chi(y<x) \sum_{k}\left|\psi_{k}(y)\right|^{2} e_{k}, \quad \widetilde{H}_{\mathrm{R}}=\sum_{y} \chi(y \geq x) \sum_{k}\left|\psi_{k}(y)\right|^{2} e_{k}
$$

From the above properties, it is clear that this is indeed a splitting and that $\left\{H_{B}, \widetilde{H}_{\mathrm{L}, \mathrm{R}}\right\}=0$, but it is not clear a-priori in which sense this splitting is similar to $H_{B}=H_{\mathrm{L}}+H_{\mathrm{R}}$ and we exhibit this now. Note first that both $H_{\mathrm{L}, \mathrm{R}}, \widetilde{H}_{\mathrm{L}, \mathrm{R}}$ are linear combinations of $p_{z} p_{w}, q_{z} q_{w}$ with $z, w \in B$. Let us call

$$
\widetilde{H}_{\mathrm{L}}-H_{\mathrm{L}}=\sum_{z, w \in B}\left(\gamma_{z, w}^{(\mathrm{L})} p_{z} p_{w}+\alpha_{z, w}^{(\mathrm{L})} q_{z} q_{w}\right), \quad \widetilde{H}_{\mathrm{R}}-H_{\mathrm{R}}=\sum_{z, w \in B}\left(\gamma_{z, w}^{(\mathrm{R})} p_{z} p_{w}+\alpha_{z, w}^{(\mathrm{R})} q_{z} q_{w}\right)
$$

with the coefficients $\gamma_{z, w}^{(\mathrm{L}, \mathrm{R})}, \alpha_{z, w}^{(\mathrm{L}, \mathrm{R})}$ chosen symmetric in $z, w$. The functions (17) are localized around $x$ in the sense that $\left|\gamma_{z, w}\right|$ typically decay exponentially in $|z-x|+|w-x|$. We will not state this in full generality because the following lemma suffices for our purposes.

Lemma 3. 1. For any $z, w,\left|\gamma_{z, w}^{(\mathrm{L}, \mathrm{R})}\right|<\mathrm{C}$ and $\left|\alpha_{z, w}^{(\mathrm{L}, \mathrm{R})}\right|<\mathrm{C}$

2. If one of $z, w$ is at the boundary of $B$, (i.e. at distance 1 of $B^{c}$ ), then

$$
E\left(\left|\gamma_{z, w}^{(\mathrm{L}, \mathrm{R})}\right|+\left|\alpha_{z, w}^{(\mathrm{L}, \mathrm{R})}\right|\right) \leq \mathrm{C} e^{-\ell / \xi}
$$


Proof. We start with (1). By combining (15) and (16), we get the explicit expression

$$
\gamma_{w, z}^{\mathrm{R}}=\frac{1}{2} \sum_{y \geq x}\left(\sum_{k}\left|\psi_{k}(y)\right|^{2} \psi_{k}(w) \psi_{k}(z)-\delta_{y, z} \delta_{z, w}\right)
$$

(for $\mathrm{L} \rightarrow \mathrm{R}$, we replace $y \geq x$ by by $y<x$ ). Using $\sum_{y}\left|\psi_{k}(y)\right|^{2}=1$ and $\sum_{k}\left|\psi_{k}(z)\right|^{2}=1$, we then estimate

$$
\left|\gamma_{w, z}^{\mathrm{R}}\right| \leq \frac{1}{2} \sum_{k}\left|\psi_{k}(w)\right|\left|\psi_{k}(z)\right|+\frac{1}{2} \leq 1
$$

and similarly for $\mathrm{R} \rightarrow \mathrm{L}$. For $\alpha$ instead of $\gamma$, we bound first $\nu_{k}^{2} \leq \mathrm{C}$ using that $\mathcal{H}_{B}$ is bounded by $\mathrm{C}$ as an operator on $l^{2}(B)$, because the distribution of $\left(\omega_{x}\right)$ has bounded support. Thereafter, the argument is analogous to that for $\gamma$.

Now to (2). For the sake of concreteness, let us take $z$ to lie at the left boundary, i.e. $z=x-\ell$ We start from (18) and we use $\left|\psi_{k}(w)\right| \leq 1$ to obtain

$$
E\left(\left|\gamma_{w, x-\ell}^{\mathrm{R}}\right|\right) \leq \sum_{y \geq x} E\left(\sum_{k}\left|\psi_{k}(y)\right|\left|\psi_{k}(x-\ell)\right|\right) \leq \mathrm{C} e^{-\ell / \xi}, \quad z=x-\ell
$$

where the crucial last inequality follows from Lemma 1 applied within $B$, and recalling that constants $\mathrm{C}$ are allowed to depend on $\xi$.

We need to get this estimate also with $\mathrm{L}$ instead of R. From

$$
\left(\widetilde{H}_{\mathrm{L}}-H_{\mathrm{L}}\right)=-\left(\widetilde{H}_{\mathrm{R}}-H_{\mathrm{R}}\right)
$$

we get

$$
\gamma_{z w}^{(\mathrm{L})}=-\gamma_{z w}^{(\mathrm{R})}
$$

which settles the desired estimate also for $\gamma_{z w}^{(\mathrm{L})}$ with $z=x-\ell$. For the case of the other boundary, i.e. $z=x+\ell$, we run an analogous argument, but starting now with $\gamma^{(\mathrm{L})}$. Finally, the argument for $\alpha$ instead of $\gamma$ proceeds analogously.

\subsubsection{Approximate solution of the Poisson equation $\mathcal{L} u_{x}=j_{x}$}

Next, we put the new left-right splitting to use to cast the local current $j_{x}$ a time-derivative $\mathcal{L} u_{x}$, up to a small correction.

Lemma 4 (localization). For $x \in G_{0}(\ell)$, there exist functions $u_{x}$ and $f_{x}$ so that

$$
\mathcal{L} u_{x}=j_{x}+f_{x}, \quad\left\langle u_{x}\right\rangle_{\beta}=0, \quad\left\langle f_{x}\right\rangle_{\beta}=0
$$

with the following properties:

1. The function $u_{x}$ has support in $B$, depends only on $\omega_{x}, x \in B$, and satisfies

$$
\left\langle u_{x}^{2}\right\rangle_{\beta} \leq C \ell^{2} .
$$

2. There is a random variable $r_{x}>0$ depending only on $\omega_{y}$ with $|x-y| \leq \ell+1$ so that,

$$
E\left(r_{x}\right) \leq \mathrm{C} \ell e^{-\ell / \xi}
$$

and, for any $x, x^{\prime} \in G_{0}(\ell)$, such that $\left|x-x^{\prime}\right|>2 \ell+2$

$$
\left\langle f_{x}^{2}\right\rangle_{\beta} \leq \mathrm{C} r_{x}^{2}, \quad\left|\left\langle f_{x} f_{x^{\prime}}\right\rangle_{\beta}\right| \leq \mathrm{C} r_{x} r_{x^{\prime}} \mathrm{e}^{-\frac{1}{\zeta}\left(\left|x-x^{\prime}\right|-(2 \ell+2)\right)}
$$


Proof. We take

$$
u_{x}=H_{L}-\widetilde{H}_{L}-\left\langle H_{L}-\widetilde{H}_{L}\right\rangle_{\beta}
$$

which satisfies all the requirements of item 1 , because of the bounds in Lemma 3. By the definition of $j_{x}$, we have that $\mathcal{L}_{B}\left(H_{L}\right)+\mathcal{L}_{B^{c}}\left(H_{L}\right)=\mathcal{L}_{B}\left(H_{L}\right)=j_{x}$ and by construction $\mathcal{L}_{B}\left(\widetilde{H}_{L}\right)=0$. Hence (19) is satisfied by

$$
f_{x}:=\left(\mathcal{L}-\mathcal{L}_{B}-\mathcal{L}_{B^{c}}\right) u_{x}=\left\{H_{\partial B}, u_{x}\right\}
$$

with

$$
H_{\partial B}=\frac{1}{2} g_{0}\left(\left(q_{x+\ell+1}-q_{x+\ell}\right)^{2}+\left(q_{x-\ell}-q_{x-\ell-1}\right)^{2}\right) .
$$

The zero-mean property $\left\langle f_{x}\right\rangle_{\beta}=0$ follows because $j_{x}$ and $\mathcal{L} w$ (for any function $w$ ) have zero mean. Using the explicit form of $u_{x}$, we get

$$
f_{x}=2 g_{0} \sum_{w \in B} p_{w}\left(\gamma_{x+\ell, w}^{(\mathrm{L})}\left(q_{x+\ell}-q_{x+\ell+1}\right)+\gamma_{x-\ell, w}^{(\mathrm{L})}\left(q_{x-\ell}-q_{x-\ell-1}\right)\right)
$$

The bound on $f_{x}$ follows now by item 2 of Lemma 3, with $r_{x}:=\sum_{z \in\{-\ell, \ell\}} \sum_{w \in B}\left|\gamma_{z, w}^{(\mathrm{L})}\right|$, by using $\left\langle q_{z}^{2} p_{w}^{2}\right\rangle_{\beta} \leq \mathrm{C}$ and the decay of correlations in Lemma 2 .

\subsection{Griffiths regions}

We now define a subset $G(\ell)$ of $G_{0}(\ell)$ where the random variable $r_{x}$ defined in Lemma 4 to bound the function $f_{x}$, is exponentially small in $\ell$ :

$$
G(\ell)=\left\{x \in G_{0}(\ell): r_{x} \leq \ell^{2} \mathrm{e}^{-\ell / \xi}\right\}
$$

Given a realization of $\left(\omega_{x}\right)_{x \geq 1}$ and $\left(\tau_{x}\right)_{x \geq 1}$, we define the strictly increasing sequence $\left(g_{i}\right)_{i \geq 1}$ as well as a sequence $\left(d_{i}\right)_{i \geq 1}$ such that

$$
\left\{g_{i}: i \geq 1\right\}=G(\ell), \quad d_{i}=g_{i+1}-g_{i}, \quad i \geq 1 .
$$

Finally, let

$$
n_{L}=\max \left\{i: g_{i} \leq L\right\} .
$$

The next lemma expresses the fact that $d_{i}$ are not too large:

Lemma 5. 1. The variables $d_{i}, d_{j}$ are independent whenever $|i-j| \geq 2 \ell+2$.

2. There exists $\ell_{0}<+\infty$ such that for any $\ell \geq \ell_{0}$, any $i \geq 1$ and any $d \geq 1$,

$$
P\left(d_{i} \geq d\right) \leq \mathrm{e}^{-d / d_{0}} \quad \text { with } \quad d_{0}=\mathrm{e}^{3 \ell \log \frac{1}{1-p}} .
$$

Proof. The first claim follows from the fact that $\left(\omega_{x}\right)_{x \geq 1}$ and $\left(\tau_{x}\right)_{x \geq 1}$ are independent sequences of i.i.d. random variables, that the event $x \in G(\ell)$ depends only on the variables $\omega_{y}, \tau_{y}$ with $|x-y| \leq \ell$, and that $d_{i} \geq 1$ for all $i \geq 1$.

For the second claim, note that $P\left(x \in G_{0}(\ell)\right) \geq(1-p)^{2 \ell+1}$ and that

$$
P\left(r_{x}>\ell^{2} e^{-\ell / \xi}\right) \leq C / \ell,
$$

by the bound (20) in Lemma 4 and the Markov inequality. Hence, for $\ell$ large enough,

$$
P(x \in G(\ell)) \geq \frac{1}{2}(1-p)^{2 \ell+1} .
$$


Since the events $x \in G(\ell)$ and $y \in G(\ell)$ are independent for $|x-y| \geq 2 \ell+2$, we obtain

$$
P\left(d_{i} \geq d\right) \leq\left(1-\frac{1}{2}(1-p)^{2 \ell+1}\right)^{d /(2 \ell+2)} \leq \exp \left(-\frac{d(1-p)^{2 \ell+1}}{4(\ell+1)}\right)
$$

which yields the claim for $\ell_{0}$ large enough.

Proof of Theorem 1. Let us fix a realization of $\left(\omega_{x}\right)_{x \geq 1}$ and $\left(\tau_{x}\right)_{x \geq 1}$. Let us fix some $t>0$ and some length $\ell=\ell(t) \in \mathbb{N}$ with a dependence on time that will be specified later on. For simplicity, let us write $G$ for $G(\ell)$. We seek an upper bound on $C(t)$ defined in (8). Given $1 \leq x<L$, let $x_{G} \in G$ be the closest point to $x$. For $x \notin G$, energy conservation implies that

$$
j_{x}=j_{x_{G}} \pm \mathcal{L}\left(\sum_{y \in\left\{x, x_{G}\right\}} h_{y}\right)
$$

where

$$
\left\{x, x_{G}\right\}=\left\{x+1, \ldots, x_{G}\right\} \quad \text { if } \quad x<x_{G}, \quad\left\{x, x_{G}\right\}=\left\{x_{G+1}, \ldots, x\right\} \quad \text { if } \quad x>x_{G}
$$

and $\pm=+$ if $x<x_{G}$, and $\pm=-$ if $x>x_{G}$. By Lemma 4 , we write $j_{x_{G}}=\mathcal{L} u_{x_{G}}-f_{x_{G}}$, and we obtain

$$
\sum_{x=1}^{L-1} j_{x}=-\sum_{x=1}^{L-1} f_{x_{G}}+\mathcal{L} \sum_{x=1}^{L-1}\left(u_{x_{G}} \pm \sum_{y \in\left\{x, x_{G}\right\}} h_{y}\right)
$$

By Cauchy-Schwarz, we estimate

$$
\begin{aligned}
& \frac{1}{2 L}\left\langle\left(\int_{0}^{t} \mathrm{~d} s \sum_{x=1}^{L-1} j_{x}(s)\right)^{2}\right\rangle_{\beta} \leq \frac{1}{L}\left\langle\left(\int_{0}^{t} \mathrm{~d} s \sum_{x=1}^{L-1} f_{x_{G}}(s)\right)^{2}\right\rangle_{\beta}+\frac{1}{L}\left\langle\left(\sum_{x=1}^{L-1} \int_{0}^{t} \mathrm{~d} s \sum_{x=1}^{L-1} \mathcal{L} v_{x}(s)\right)^{2}\right\rangle_{\beta} \\
& =: \quad I_{1}(L, t) \quad+\quad I_{2}(L, t)
\end{aligned}
$$

where we abbreviated

$$
v_{x}=u_{x_{G}} \pm \sum_{y \in\left\{x, x_{G}\right\}} h_{y}
$$

To prove Theorem 1, we establish almost sure bounds on $\lim _{L} I_{j}(L, t)$, for $j=1,2$.

We start with $I_{1}(t)$. By Cauchy-Schwarz and stationarity, we have for any $w$,

$$
\left\langle\left(\int_{0}^{t} \mathrm{~d} s w(s)\right)^{2}\right\rangle_{\beta} \leq t^{2}\left\langle w^{2}\right\rangle_{\beta}
$$

Taking $w=\sum_{x=1}^{L-1} f_{x_{G}}$, this yields here

$$
I_{1}(L, t) \leq \frac{t^{2}}{L}\left\langle\left(\sum_{x=1}^{L} f_{x_{G}}\right)^{2}\right\rangle_{\beta}=\frac{t^{2}}{L} \sum_{x, y \in \mathbb{N}_{L}}\left\langle f_{x_{G}} f_{y_{G}}\right\rangle_{\beta}
$$

Using the variables $g_{i}, d_{i}$ defined in (22), we get then 


$$
\sum_{x, y \in \mathbb{N}_{L}}\left\langle f_{x_{G}} f_{y_{G}}\right\rangle_{\beta} \leq \sum_{1 \leq i, j \leq n_{L}}\left(d_{i}+d_{i+1}\right)\left(d_{j}+d_{j+1}\right)\left|\left\langle f_{g_{i}} f_{g_{j}}\right\rangle_{\beta}\right|
$$

By the definition (21) of the set $G$, the bounds of Lemma 4 and independence of the variables $r_{x}, r_{y}$ for $|x-y| \geq 2 \ell+2$, we get

$$
\sum_{x, y \in \mathbb{N}_{L}}\left\langle f_{x_{G}} f_{y_{G}}\right\rangle_{\beta} \leq \mathrm{C} \ell^{5} \mathrm{e}^{-2 \ell / \xi} \sum_{1 \leq i, j \leq n_{L}} \mathrm{e}^{-\frac{1}{\zeta}|i-j|} d_{i} d_{j}
$$

Estimating $d_{i} d_{j} \leq \frac{1}{2}\left(d_{i}^{2}+d_{j}^{2}\right)$, we hence get

$$
I_{1}(L, t) \leq \mathrm{C} t^{2} \ell^{5} \mathrm{e}^{-2 \ell / \xi} \frac{1}{L} \sum_{i=1}^{n_{L}} d_{i}^{2}
$$

By Lemma 5, the variables $d_{i}^{2}$ have finite moments and they have finite-range correlations. This suffices to establish a strong law of large numbers for the sequence $d_{i}^{2}$. Moreover, $E\left(d_{i}^{2}\right) \leq \mathrm{C} d_{0}^{2}$ and hence we obtain the almost sure bound:

$$
I_{1}(t):=\varlimsup_{L \rightarrow \infty} I_{1}(L, t) \leq \mathrm{C} t^{2} \ell^{5} \mathrm{e}^{b_{1} \ell}, \quad b_{1}=-\frac{2}{\xi}+6 \log \frac{1}{1-p} .
$$

Next, we consider the limit $t \rightarrow \infty$. We set, for some $a>0$ to be determined later,

$$
\ell(t)=a \log t
$$

and we obtain

$$
I_{1}(t)=\mathcal{O}\left((\log t)^{5} t^{2+a b_{1}}\right), \quad t \rightarrow \infty
$$

We now move to $I_{2}(L, t)$, as defined in $(25)$. For any $v$ in the domain of $\mathcal{L}$,

$$
\left\langle\left(\int_{0}^{t} \mathrm{~d} s \mathcal{L} v(s)\right)^{2}\right\rangle_{\beta}=\left\langle(\tilde{v}(t)-\tilde{v}(0))^{2}\right\rangle_{\beta} \leq 2\left\langle\tilde{v}^{2}\right\rangle_{\beta}
$$

with $\tilde{v}=v-\langle v\rangle_{\beta}$. We use this with $v=\sum_{x} v_{x}$ and $v_{x}$ as defined in (26). This yields

$$
\begin{aligned}
& I_{2}(L, t) \leq \frac{2}{L}\left\langle\left(\sum_{x=1}^{L-1}\left(u_{x_{G}} \pm \sum_{y \in\left\{x, x_{G}\right\}} \tilde{h}_{y}\right)\right)^{2}\right\rangle_{\beta} \\
& \leq \frac{4}{L} \sum_{x, y \in \mathbb{N}_{L}}\left|\left\langle u_{x_{G}} u_{y_{G}}\right\rangle_{\beta}\right|+\frac{4}{L} \sum_{x, y \in \mathbb{N}_{L}} \sum_{\substack{x^{\prime} \in\left\{x, x_{G}\right\}, y^{\prime} \in\left\{y, y_{G}\right\}}}\left|\left\langle\tilde{h}_{x^{\prime}} \tilde{h}_{y^{\prime}}\right\rangle_{\beta}\right| \text {. }
\end{aligned}
$$

Thanks to the decay of correlations in Lemma 2 and the properties of $u_{x_{G}}$ stated in Lemma 4 , we have

$$
\left|\left\langle u_{x_{G}} u_{y_{G}}\right\rangle_{\beta}\right| \leq \mathrm{C} \ell^{4} \mathrm{e}^{-\left|x_{G}-y_{G}\right| / \zeta}, \quad\left|\left\langle\tilde{h}_{x^{\prime}} \tilde{h}_{y^{\prime}}\right\rangle_{\beta}\right| \leq \mathrm{Ce}^{-\left|x^{\prime}-y^{\prime}\right| / \zeta} .
$$

Just as for $I_{1}(L, t)$, we estimate the number of $x$ such that $x_{G}=g_{i}$ by $d_{i}+d_{i+1}$ and we obtain

$$
I_{2}(L, t) \leq \frac{\mathrm{C} \ell^{4}}{L} \sum_{1 \leq i, j \leq n_{L}} d_{i} d_{j} \mathrm{e}^{-|i-j| / \zeta}+\frac{\mathrm{C}}{L} \sum_{1 \leq i, j \leq n_{L}} d_{i}^{2} d_{j}^{2} \mathrm{e}^{-|i-j| / \zeta} \leq \frac{\mathrm{C} \ell^{4}}{L} \sum_{i=1}^{n_{L}} d_{i}^{4}
$$


where we used $d_{i}^{m} d_{j}^{m} \leq d_{i}^{m}+d_{j}^{m}$ and $d_{i} \leq d_{i}^{2}$ since $d_{i} \geq 1$. In the limit $L \rightarrow \infty$, we can again invoke the strong law of large numbers and we obain the almost sure bound

$$
I_{2}(t):=\varlimsup_{L \rightarrow \infty} I_{2}(L, t) \leq \mathrm{C} \ell^{4} \mathrm{e}^{b_{2} \ell}, \quad b_{2}=12 \log \frac{1}{1-p}
$$

We see hence that, for $\ell=\ell(t)$ given by $(27), I_{2}(t)=O\left((\log t)^{4} t^{a b_{2}}\right)$.

Adding the contributions of $I_{1}, I_{2}$, we conclude that

$$
C(t) \leq C(\log t)^{5}\left(t^{2+a b_{1}}+t^{a b_{2}}\right)
$$

The optimal $a$ is found by equating the two powers: $2+a b_{1}=a b_{2}$, yielding

$$
a=\frac{1}{v+1 / \xi}, \quad v=3 \log \left(\frac{1}{1-p}\right) .
$$

This yields the claims of the theorem.

\section{Proof of Theorem 2}

This proof is entirely analogous to that of Theorem 1 but we still repeat the initial steps, because there are some superficial differences.

We let constants $\mathrm{C}$ depend on $J, g, \mu$ and the distribution of $\omega_{x}$.

\subsection{Observables and Gibbs state}

Any observable $a$ can be expanded in a unique way as a linear combination of normally ordered monomials

$$
c_{Y}^{\dagger}=c_{y_{1}}^{\dagger} \ldots c_{y_{p}}^{\dagger}, \quad c_{Y^{\prime}}=c_{y_{p^{\prime}}^{\prime}} \ldots c_{y_{1}^{\prime}}, \quad p, p^{\prime} \geq 0 .
$$

Here $Y, Y^{\prime}$ are shorthand for finite tuples of $y$-coordinates. $p=0$ means that there are no annihilation operators, and analogously for $p^{\prime}$, and hence $p=p^{\prime}=0$ is the identity. Hence we can write

$$
a=\sum_{Y, Y^{\prime}} a\left(Y, Y^{\prime}\right) c_{Y^{\prime}}^{\dagger} c_{Y}
$$

and we define the support of $a$ as $\operatorname{supp}(a)=Y \cup Y^{\prime}$. In general, let $H_{X}$ be the Hamiltonian restricted to a finite set $X \subset \mathbb{N}$, i.e. retaining only terms in (9) whose support is in $X$. Similarly, we define the restriction $N_{X}=\sum_{x \in X} n_{x}$ and we note that $\left[H_{X}, N_{X}\right]=0$. Finally, we also define the restriction $\widetilde{\mathcal{H}}_{X}=\sum_{x, y \in X} \widetilde{\mathcal{H}}_{x, y}$ of $\widetilde{\mathcal{H}}$. As an application of these definition, we check that, if, as will be assumed in the next section, the variables $\tau_{y}=0$ for $y \in B$ with $B$ a stretch of sites, then the restriction $H_{B}$ is the second quantization of $\widetilde{\mathcal{H}}$;

$$
H_{B}=\sum_{\{x, x+1\} \in B} J\left(c_{x+1 c_{x}}^{\dagger}+c_{x}^{\dagger} c_{x+1}\right)+\sum_{x \in B} \omega_{x} n_{x}=\sum_{x, x^{\prime}} c_{x^{\prime}}^{\dagger}\left(\widetilde{\mathcal{H}}_{B}\right)_{x, x^{\prime}} c_{x}
$$

Let us comment on the state $\langle\cdot\rangle_{\mu}$ defined in (11). This state is very easy to work with as it is the analogue of a product state on the lattice. Since the density matrix factorizes, $\mathrm{e}^{-\mu N}=\prod_{x} \mathrm{e}^{-\mu n_{x}}$, one easily establishes 
Lemma 6. 1. Let a be an observable of the form $a=a\left(Y, Y^{\prime}\right) c_{Y}^{\dagger} c_{Y^{\prime}}$, then

$$
\langle a\rangle_{\mu} \leq\|a\| \leq\left|a\left(Y, Y^{\prime}\right)\right|
$$

2. Whenever $\operatorname{supp}(a) \cap \operatorname{supp}(b)=\emptyset$, then

$$
\langle a b\rangle_{\mu}=0
$$

Here, the $\|\cdot\|$ stands for the usual operator norm.

\subsection{Splitting of the quadratic Hamiltonian}

We now exploit the fact that the system is an Anderson insulator in the regions where the anharmonic potential is absent, i.e. where $\tau_{x}=0$. The upshot is Lemma 7 . Throughout this section, we fix a realization of $\left(\tau_{x}\right)_{x \geq 1}$. Given $\ell \in \mathbb{N}$, let

$$
G_{0}(\ell)=\left\{x \in \mathbb{N}: \tau_{y}=0 \text { for all } y \in \mathbb{N} \text { s.t. }|y-x| \leq \ell\right\} .
$$

We also fix an element $x \in G_{0}(\ell)$ and we denote $B=\{x-\ell, \ldots, x+\ell\}$, such that $\tau_{y}=0$ for $y \in B$. Until the end of this section, the expectation $E$ is assumed to be conditioned on $\tau_{y}=0, y \in B$ and we do not repeat this.

We first consider an obvious splitting of $N_{B}$ into a left (L) and right (R) part with respect to the midpoint $x$ :

$$
N_{B}=N_{\mathrm{L}}+N_{\mathrm{R}}, \quad N_{\mathrm{L}}:=N_{\{y \in B: y<x\}}, \quad N_{\mathrm{R}}:=N_{\{y \in B: y \geq x\}}
$$

The observables $N_{\mathrm{L}}, N_{\mathrm{R}}$ do not commute with $H_{B}$ in general, corresponding to the fact that particles can be transported. Our aim in this section is to find a modified left-right splitting $N_{B}=\widetilde{N}_{\mathrm{L}}+\widetilde{N}_{\mathrm{R}}$ which does satisfy $\left[H_{B}, \widetilde{N}_{\mathrm{L}, \mathrm{R}}\right]=0$, reflecting the spatial localization of energy.

We now recall the $H_{B}$ is quadratic in the $c_{y}, c_{y}^{\dagger}$-operators, which allows for an explicit analysis. As before, we let $\left(\psi_{k}\right)_{k \in \mathcal{I}}$ be an orthonormal basis of eigenvectors of $\widetilde{\mathcal{H}}_{B}$, with $\mathcal{I}$ an index set, $|\mathcal{I}|=|B|$. We define the eigenmode operators

$$
c_{k}=\sum_{k} \psi_{k}(x) c_{x}, \quad c_{k}^{\dagger}=\sum_{k} \psi_{k}(x) c_{x}^{\dagger}, \quad n_{k}=c_{k}^{\dagger} c_{k}
$$

Their two useful properties are

1. $\left[H_{B}, n_{k}\right]=0$

2. $N=\sum_{x} n_{x}=\sum_{k} n_{k}$

The second property follows immediately from the Plancherel equality, and the first is an expression of the fact that $H_{B}$ is the second quantization of $\widetilde{\mathcal{H}}_{B}$, as exhibited in (29). The splitting $N_{B}=\widetilde{N}_{\mathrm{L}}+\widetilde{N}_{\mathrm{R}}$ that we propose is defined by

$$
\tilde{N}_{\mathrm{L}}=\sum_{y<x} \sum_{k}\left|\psi_{k}(y)\right|^{2} n_{k}, \quad \tilde{N}_{\mathrm{R}}=\sum_{y \geq x} \sum_{k}\left|\psi_{k}(y)\right|^{2} n_{k}
$$


From the above properties, it is clear that this is indeed a splitting and that $\left\{H_{B}, \widetilde{N}_{\mathrm{L}, \mathrm{R}}\right\}=0$, but it is not clear a-priori in which sense this splitting is similar to $N_{B}=N_{\mathrm{L}}+N_{\mathrm{R}}$ and we exhibit this now. Note first that both $N_{\mathrm{L}, \mathrm{R}}, \widetilde{N}_{\mathrm{L}, \mathrm{R}}$ are linear combinations of $c_{z}^{\dagger} c_{w}$ with $z, w \in B$. Let us call

$$
N_{\mathrm{L}}-\tilde{N}_{\mathrm{L}}=\sum_{z, w \in B} \gamma_{z, w}^{(\mathrm{L})} c_{z}^{\dagger} c_{w}, \quad N_{\mathrm{R}}-\tilde{N}_{\mathrm{R}}=\sum_{z, w \in B} \gamma_{z, w}^{(\mathrm{R})} c_{z}^{\dagger} c_{w}
$$

The functions (32) are small in the sense that the coefficients $\gamma_{z, w}$ typically decay exponentially in $|z-x|+|w-x|$. We will not state this in full generality because the following lemma suffices for our purposes.

Lemma 7. 1. For any $z, w,\left|\gamma_{z, w}^{(\mathrm{L}, \mathrm{R})}\right|<\mathrm{C}$.

2. If one of $z, w$ is at the boundary of $B$, (i.e. at distance 1 of $\left.B^{c}\right)$, then

$$
E\left(\left|\gamma_{z, w}^{(\mathrm{L}, \mathrm{R}}\right|\right) \leq \mathrm{C} e^{-\ell / \xi}
$$

Proof. From the above we derive an explicit expression for $\gamma_{w, z}^{\mathrm{R}, \mathrm{R}}$ and this expression is identical to the one for the classical anharmonic case, i.e. equation (18) holds without any change. We can therefore copy line per line the proof of Lemma 3.

Next, we put the new left-right splitting to use to cast the local current $j_{x}$ a time-derivative $j_{x}=\mathcal{L} u_{x}$, up to a small correction.

Lemma 8 (localization). For $x \in G_{0}(\ell)$, there exist observables $u_{x}$ and $f_{x}$ so that

$$
\mathcal{L} u_{x}=j_{x}+f_{x}, \quad\left\langle u_{x}\right\rangle_{\mu}=0 ;, \quad\left\langle f_{x}\right\rangle_{\mu}=0
$$

with the following properties:

1. The observable $u_{x}$ has support in $B$, depends only on $\omega_{x}, x \in B$, and satisfies

$$
\left\langle u_{x}^{2}\right\rangle_{\mu} \leq \mathrm{C} \ell^{2}
$$

2. There is a random variable $r_{x}>0$ depending only on $\omega_{y}$ with $|x-y| \leq \ell+1$ so that,

$$
E\left(r_{x}\right) \leq \mathrm{C} \ell e^{-\ell / \xi}
$$

and, for any $x, x^{\prime} \in G_{0}(\ell)$, such that $\left|x-x^{\prime}\right|>2 \ell+2$

$$
\left\langle f_{x}^{2}\right\rangle_{\mu} \leq \mathrm{C} r_{x}^{2}, \quad\left\langle f_{x} f_{x^{\prime}}\right\rangle_{\mu}=0
$$

Proof. We take

$$
u_{x}=N_{L}-\tilde{N}_{L}-\left\langle N_{L}-\tilde{N}_{L}\right\rangle_{\beta}
$$

which satisfies all the requirements of (1), because of the bounds in Lemma 7. By the definition of $j_{x}$, we have that $\mathcal{L}_{B}\left(N_{\mathrm{L}}\right)+\mathcal{L}_{B^{c}}\left(N_{\mathrm{L}}\right)=\mathcal{L}_{B}\left(N_{\mathrm{L}}\right)=j_{x}$ and by construction $\mathcal{L}_{B}\left(\widetilde{N}_{\mathrm{L}}\right)=0$. Hence (33) is satisfied by

$$
f_{x}:=\left(\mathcal{L}-\mathcal{L}_{B}-\mathcal{L}_{B^{c}}\right) u_{x}=\mathrm{i}\left[H_{\partial B}, u_{x}\right]
$$


with

$$
H_{\partial B}=\sum_{y \in\{x-\ell-1, x+\ell\}} J\left(c_{y}^{\dagger} c_{y+1}+h c\right)+g \tau_{y} n_{y} n_{y+1}
$$

leading also to the correct support properties. To check that $\left\langle f_{x}\right\rangle_{\mu}=0$, we use that $\langle\mathcal{L} w\rangle_{\mu}=0$ for any observable $w$ and that $\left\langle j_{x}\right\rangle_{\mu}=0$, see comment in Section 2.2.1. Using the explicit form of $u_{x}$, we get

$$
f_{x}=\sum_{z \in\{-\ell, \ell\}} \sum_{w \in B} \gamma_{z, w}\left[H_{\partial B}, c_{z}^{\dagger} c_{w}\right]
$$

Putting $r_{x}:=\sum_{z \in\{-\ell, \ell\}} \sum_{w \in B}\left|\gamma_{z, w}\right|$, the claims follow by Lemma 32$)$, the fact that $\left\|H_{\partial B}\right\| \leq \mathrm{C}$, and the product state property in Lemma 6.

\subsection{Griffiths regions}

The rest of the argument proceeds just as in the classical case, in Section 3.3. In particular, the definition of the set $G(\ell)$ and its main properties, i.e. Lemma 5, is taken over without any change. Then, the reasoning in the rest of the proof for the classical anharmonic chain relies entirely on the representation (23) for $x \notin G(\ell)$

$$
j_{x}=j_{x_{G}} \pm \mathcal{L}\left(\sum_{y \in\left\{x, x_{G}\right\}} h_{y}\right)
$$

(with the set $\left\{x, x_{G}\right\}$ defined below (23)). For the fermionic chain, we simply change this to

$$
j_{x}=j_{x_{G}} \pm \mathcal{L}\left(\sum_{y \in\left\{x, x_{G}\right\}} n_{y}\right)
$$

The role of Lemma 2 is played here by Lemma 6 , and hence we can replace the correlation length $\zeta$ by 0 .

Acknowledgements. We are most grateful to David A. Huse and J. L. Lebowitz who suggested the study of the disordered models introduced in this paper. The work of F. H. and S.O. was partially supported by the grant ANR-15-CE40-0020-01 LSD of the French National Research Agency (ANR). F. H. acknowledges also the support of the ANR under grant ANR-14-CE250011 EDNHS. W. D. R. acknowledges the support of the Flemish Research Fund FWO under grants G098919N and G076216N, and the support of KULeuven University under internal grant C14/16/062.

\section{References}

[1] H. Abdul-Rahman, B. Nachtergaele, R. Sims, and G. Stolz. Localization properties of the disordered XY spin chain: A review of mathematical results with an eye toward many-body localization. Annalen der Physik, 529(7):1600280, 2017. 
[2] K. Agarwal, E. Altman, E. Demler, S. Gopalakrishnan, D. A. Huse, and M. Knap. Rareregion effects and dynamics near the many-body localization transition. Annalen der Physik, 529(7):1600326, 2017. 1600326.

[3] K. Agarwal, S. Gopalakrishnan, M. Knap, M. Müller, and E. Demler. Anomalous diffusion and Griffiths effects near the many-body localization transition. Physical Review Letters, 114(16):160401, 2015.

[4] R. Alicki and M. Fannes. Quantum dynamical systems. Oxford university press, 2001.

[5] E. Altman and R. Vosk. Universal dynamics and renormalization in many-body-localized systems. Annual Review of Condensed Matter Physics, 6(1):383-409, 2015.

[6] P. W. Anderson. Absence of diffusion in certain random lattices. Physical Review, 109:1492$1505,1958$.

[7] J. H. Bardarson, F. Pollmann, U. Schneider, and S. Sondhi, editors. Special Issue: ManyBody Localization, volume 529(7) of Annalen der Physik. John Wiley \& Sons, 2017.

[8] D. Basko. Weak chaos in the disordered nonlinear Schrödinger chain: destruction of Anderson localization by Arnold diffusion. Annals of Physics, 326(7):1577-1655, 2011.

[9] D. M. Basko, I. L. Aleiner, and B. L. Altshuler. Metalinsulator transition in a weakly interacting many-electron system with localized single-particle states. Annals of Physics, 321(5):1126-1205, 2006.

[10] C. Bernardin and F. Huveneers. Small perturbation of a disordered harmonic chain by a noise and an anharmonic potential. Probability Theory and Related Fields, 157(1):301-331, 2013.

[11] T. Bodineau and B. Helffer. Correlations, Spectral Gap, and Log-Sobolev Inequalities for Unbounded Spin Systems. In R. Weikard and G. Weinstein, editors, Studies in Advanced Mathematics, Differential Equations and Mathematical Physics, volume 16, pages 51-66. AMS/IP, 2000.

[12] A. Casher and J. L. J. L. Lebowitz. Heat Flow in Regular and Disordered Harmonic Chains. Journal of Mathematical Physics, 12(8):1701-1711, 1971.

[13] H. L. Cycon, R. G. Froese, W. Kirsch, and B Simon. Schrödinger Operators with Applications to Quantum Mechanics and Global Geometry. Springer-Verlag, 1987.

[14] D. Damanik. A Short Course on One-Dimensional Random Schrödinger Operators. arXiv e-prints, arXiv:1107.1094, 2011.

[15] W. De Roeck and F. Huveneers. Stability and instability towards delocalization in manybody localization systems. Physical Review B, 95:155129, 2017.

[16] W. De Roeck and F. Huveneers. Glassy dynamics in strongly anharmonic chains of oscillators. arXiv e-prints, page arXiv:1904.07742, 2019. 
[17] Giuseppe De Tomasi, Soumya Bera, Antonello Scardicchio, and Ivan M Khaymovich. Subdiffusion in the anderson model on random regular graph. arXiv preprint arXiv:1908.11388, 2019 .

[18] A. Dhar and J. L. Lebowitz. Effect of Phonon-Phonon Interactions on Localization. Physical Review Letters, 100:134301, 2008.

[19] R. Ducatez. A forward-backward random process for the spectrum of 1D Anderson operators. arXiv e-prints, arXiv:1711.11302, 2017.

[20] L. Fleishman and P. W. Anderson. Interactions and the Anderson transition. Physical Review B, 21(6):2366, 1980.

[21] J. Fröhlich and T. Spencer. Absence of diffusion in the Anderson tight binding model for large disorder or low energy. Communications in Mathematical Physics, 88(2):151-184, 1983.

[22] I. Ya. Gol'dshtein, S. A. Molchanov, and L. A. Pastur. A pure point spectrum of the stochastic one-dimensional Schrödinger operator. Functional Analysis and Its Applications, 11(1):1-8, 1977.

[23] S. Gopalakrishnan, K. Agarwal, E. Demler, D. Huse, and M. Knap. Griffiths effects and slow dynamics in nearly many-body localized systems. Physical Review B, 93(13):134206, 2016 .

[24] I. Gornyi, A. Mirlin, and D. Polyakov. Interacting electrons in disordered wires: Anderson localization and low-T transport. Physical Review Letters, 95(20):206603, 2005.

[25] B. Helffer. Remarks on Decay of Correlations and Witten Laplacians Brascamp-Lieb Inequalities and Semiclassical Limit. Journal of Functional Analysis, 155(2):571-586, 1998.

[26] B. Helffer. Remarks on decay of correlations and Witten Laplacians III. Application to logarithmic Sobolev inequalities. Annales de l'Institut Henri Poincare, section B, 35(4):483508, 1999.

[27] J. Imbrie. On many-body localization for quantum spin chains. Journal of Statistical Physics, 163(5):998-1048, 2016.

[28] M. Kozarzewski, P. Prelovšek, and M. Mierzejewski. Spin subdiffusion in the disordered Hubbard chain. Physical Review Letters, 120(24):246602, 2018.

[29] H. Kunz and B. Souillard. Sur le spectre des opérateurs aux différences finies aléatoires. Communications in Mathematical Physics, 78(2):201-246, 1980.

[30] S. Lepri, editor. Thermal transport in low dimensions: from statistical physics to nanoscale heat transfer, volume 921 of Lecture Notes in Physics. Springer, 2016.

[31] Yevgeny Bar Lev, Guy Cohen, and David R Reichman. Absence of diffusion in an interacting system of spinless fermions on a one-dimensional disordered lattice. Physical review letters, 114(10):100601, 2015. 
[32] D. J. Luitz and Y. Bar Lev. Anomalous Thermalization in Ergodic Systems. Physical Review Letters, 117:170404, Oct 2016.

[33] D. J. Luitz and Y. Bar Lev. The ergodic side of the many-body localization transition. Annalen der Physik, 529(7):1600350, 2017.

[34] D. J. Luitz, F. Huveneers, and W. De Roeck. How a Small Quantum Bath Can Thermalize Long Localized Chains. Physical Review Letters, 119:150602, 2017.

[35] D. J. Luitz, N. Laflorencie, and F. Alet. Extended slow dynamical regime close to the many-body localization transition. Physical Review B, 93(6):060201, 2016.

[36] Juan Jose Mendoza-Arenas, M Žnidarič, Vipin Kerala Varma, John Goold, Stephen R Clark, and Antonello Scardicchio. Asymmetry in energy versus spin transport in certain interacting disordered systems. Physical Review B, 99(9):094435, 2019.

[37] M. Mulansky, K. Ahnert, A. Pikovsky, and D. Shepelyansky. Dynamical thermalization of disordered nonlinear lattices. Physical Review E, 80:056212, 2009.

[38] B. Nachtergaele and J. Reschke. Slow propagation in some disordered quantum spin chains. arXiv e-prints, page arXiv:1906.10167, 2019.

[39] V. Oganesyan and D. A. Huse. Localization of interacting fermions at high temperature. Physical Review B, 75:155111, 2007.

[40] V. Oganesyan, A. Pal, and D. Huse. Energy transport in disordered classical spin chains. Physical Review B, 80(11):115104, 2009.

[41] A. C. Potter, R. Vasseur, and S. A. Parameswaran. Universal properties of many-body delocalization transitions. Physical Review X, 5(3):031033, 2015.

[42] Sthitadhi Roy, Yevgeny Bar Lev, and David J Luitz. Anomalous thermalization and transport in disordered interacting floquet systems. Physical Review B, 98(6):060201, 2018.

[43] R. J. Rubin and W. L. Greer. Abnormal Lattice Thermal Conductivity of a OneDimensional, Harmonic, Isotopically Disordered Crystal. Journal of Mathematical Physics, 12(8):1686-1701, 1971.

[44] Maximilian Schulz, Scott Richard Taylor, Christopher Andrew Hooley, and Antonello Scardicchio. Energy transport in a disordered spin chain with broken U(1) symmetry: Diffusion, subdiffusion, and many-body localization. Physical Review B, 98(18):180201, 2018.

[45] M. Serbyn, Z. Papić, and D. Abanin. Local conservation laws and the structure of the many-body localized states. Physical Review Letters, 111(12):127201, 2013.

[46] M. Žnidarič, A. Scardicchio, and V. K. Varma. Diffusive and subdiffusive spin transport in the ergodic phase of a many-body localizable system. Physical Review Letters, 117(4):040601, 2016. 